FORMATION Formation emploi

Revue française de sciences sociales

139 | Juillet-Septembre 2017

De l'autonomie dans les parcours professionnels

\title{
Parcours de travailleurs handicapés : autonomies contrariées et désajustements institutionnels
}

Disabled workers' life courses : impeded autonomies and institutional

discrepancies

Laufbahn behinderter Berufstätiger : verhinderte Autonomie und institutionelle Fehlanpassungen

Itinerarios de trabajadores discapacitados : autonomías contrariadas y

desajustes institucionales

\section{Louis Bertrand}

\section{CpenEdition}

Journals

Édition électronique

URL : http://journals.openedition.org/formationemploi/5134

DOI : 10.4000/formationemploi.5134

ISSN : 2107-0946

Éditeur

La Documentation française

Édition imprimée

Date de publication : 15 octobre 2017

Pagination : $33-50$

ISSN : 0759-6340

Référence électronique

Louis Bertrand, «Parcours de travailleurs handicapés : autonomies contrariées et désajustements institutionnels », Formation emploi [En ligne], 139 | Juillet-Septembre 2017, mis en ligne le 15 octobre 2017, consulté le 30 octobre 2020. URL : http://journals.openedition.org/formationemploi/5134; DOI : https://doi.org/10.4000/formationemploi.5134 


\title{
Parcours de travailleurs handicapés : autonomies contrariées et désajustements institutionnels
}

\author{
LOUIS BERTRAND \\ Sociologue. Post-doctorant, Institut national de la santé et de la recherche médicale (Inserm) - \\ Centre de recherche médecine, sciences, santé, santé mentale, société (Cermes3), Villejuif
}

Résumé

Parcours de travailleurs handicapés : autonomies contrariées et désajustements institutionnels

À l'instar d'autres politiques sociales, les politiques françaises du handicap insistent sur l'autonomie de décision des personnes handicapées, en se basant sur l'expression de leur " projet de vie ". Cet article s'intéresse aux mesures d'orientation concernant les " travailleurs handicapés ». Quelle est l'influence de ce statut sur leurs parcours ? Comment les professionnels de l'insertion agissent-ils sur les projets des demandeurs ? Nous montrons, d'une part, que l'autonomie des travailleurs handicapés est difficile à prendre en compte par les professionnels de l'insertion et, d'autre part, que bien des demandeurs n'ont pas de projet professionnel précis et s'en remettent alors aux préconisations des services d'orientation.

Mots clés : travailleur handicapé ; politique sociale ; orientation tout au long de la vie accompagnement professionnel ; cheminement professionnel ; récit de vie ; projet professionnel; reconversion professionnelle; accès a la FPC

Abstract

\section{Disabled workers' life courses : impeded autonomies and institutional discrepancies}

As other social policies, French disability policies stress on the decisional autonomy of disabled peoples, as expressed in their written " life project". This article deals with the work transition schemes for "disabled workers". How does this status affects their life course? How does the professionals influence the projects of the clients? We show that on one side for the institutions the autonomy of the disabled workers is difficult to take in account and on the other side that a lot of clients don't have a clear professional project and rely on the requirements of the transition services.

Keywords: disabled worker ; social policy; lifelong guidance ; coaching; occupational paths ; life story; career project ; occupational retraining ; access to CVT

Journal of Economic Literature: J 14 ; J 24

Traduction : Auteur. 
La notion d'autonomie travaille le champ intellectuel et politique depuis maintenant plusieurs décennies, qu'on y voit une mutation du capitalisme, un symptôme de l'individualisation des sociétés ou une injonction morale et politique (Jouan, Laugier, 2009). Pour caractériser ces conceptions de l'autonomie et leurs ambiguïtés, l'étude de certaines fractions de la population considérées comme dominées, vulnérables ou discriminées et de leur prise en charge publique paraît fructueuse (Bresson, 2004). D'une certaine manière, l'autonomie de ces personnes fait problème, qu'elle soit entravée ou déficitaire, qu'elle soit perçue comme dangereuse ou comme insuffisante ${ }^{1}$.

Dans le champ des politiques sociales, la montée en puissance du thème de l'autonomie des demandeurs s'inscrit dans un mouvement d'individualisation des prises en charge et d'apparition des politiques d'insertion. L'accompagnement des personnes est censé se centrer sur leur projet, et la relation entre bénéficiaires et institutions et ses objectifs sont souvent formalisés dans un " contrat ».

En France, ces "politiques d'insertion » ne visent pas seulement des objectifs de réinsertion professionnelle, mais également une insertion sociale (Barbier, 2002 ; Morel, 2000). Concernant l'étude des institutions de socialisation, on peut différencier deux approches. La première souligne le poids des institutions et leur pouvoir structurel. François Dubet (2002) a retracé l'histoire des "programmes institutionnels", dont il évoque à la fois l'héritage et le déclin. Une deuxième approche insiste sur un éclatement des institutions au profit d'usagers définissant librement les buts de leur accompagnement (Soulet, 2005 ; Rosanvallon, 1995). Schématiquement, il y aurait, d'un côté, la persistance d'une emprise institutionnelle et de l'autre, le retrait des institutions au profit de l'autonomie d'usagers "mis au centre ". On peut y voir un lointain écho des descriptions "misérabilistes" et "populistes» des mondes populaires analysées par Claude Grignon et Jean-Pierre Passeron (1989). Rappelons que ces auteurs appelaient à un dépassement des apories de ces deux approches par une alternance des points de vue sur "le populaire».

Depuis la fin des années 1990, plusieurs études marquantes ont étudié empiriquement les évolutions des politiques sociales : études des relations au guichet (Dubois, 2010 ; Weller, 1999 ; Siblot, 2006) et des magistratures sociales attribuant des droits au cas par cas (Astier, 1997 ; Lima, 2013), parcours des usagers des politiques d'insertion (Duvoux, 2009). Chacun à leur manière, ces travaux montrent les libertés et les contraintes qui pèsent sur les acteurs en présence, les stratégies adoptées pour gagner en marge de manœuvre et leurs limites. Toutefois, ces recherches, hormis celle de Yasmine Siblot (op. cit.), ont rarement investigué les deux côtés du guichet, privilégiant soit le point de vue des «street level bureaucrats" (Lipsky, 1980), soit celui des usagers.

1. L'auteur remercie les coordinatrices du numéro, les relecteurs anonymes et la rédaction de la revue qui, par leurs lectures et leurs conseils, ont contribué à améliorer cet article. 


\section{Encadré 1 : L'insertion professionnelle des travailleurs handicapés dans les politiques du handicap}

Les politiques visant l'insertion professionnelle des travailleurs handicapés sont principalement organisées par les lois de 1975, 1987 et 2005 (1). Les travailleurs handicapés sont amenés à travailler soit en milieu " ordinaire » (marché du travail classique), soit en milieu « protégé », principalement dans les ESAT (2).

En milieu ordinaire, « l'obligation d'embauche de travailleurs handicapés » (OETH) impose l'embauche d'un quota de $6 \%$ de ces travailleurs aux établissements de plus de vingt salariés. Il est possible de répondre à cette obligation par l'embauche directe de travailleurs handicapés, par la sous-traitance au secteur protégé ou par la cotisation à un fonds pour l'insertion professionnelle de travailleurs handicapés (3).

Sont reconnus comme travailleurs handicapés les titulaires de diverses prestations comme I'AAH (Allocation aux Adultes Handicapés) ou la carte d'invalidité, mais les personnes éligibles sont principalement les titulaires d'une " reconnaissance de la qualité de travailleur handicapé » (RQTH). Cette mesure, doit être demandée auprès des MDPH (Maisons Départementales des Personnes Handicapées), instaurées par la loi de 2005 à la suite des anciennes Cotorep (4).

Les réformes des politiques du handicap affectent le traitement des demandes de RQTH. La MDPH est censée mener une évaluation globale de la situation, sur la base d'un " projet de vie » exprimé par le demandeur. Devant une demande de RQTH, la MDPH doit se prononcer sur une " orientation professionnelle » des demandeurs. Elle doit également évaluer l'employabilité des demandeurs de l'AAH, que ceux-ci aient demandé la RQTH ou pas. Enfin, elle doit réaliser cette évaluation en quatre mois, ce qui représente une forte contrainte de temps dans les MDPH que nous avons observées - où le temps de réponse est plutôt de six mois (5).

Pour autant, la MDPH a souvent peu d'éléments pour apporter une réponse " globale » et « individualisée » en matière d'insertion professionnelle. Le panel de ses réponses reste limité : octroi ou non d'une RQTH (dont elle peut moduler la durée), orientation vers le milieu ordinaire, le milieu protégé, ou en formation adaptée (notamment dans les « centres de rééducation professionnelle »). Ces orientations relèvent formellement de l'« orientation professionnelle ». Les orientations en milieu protégé ou en formation adaptée sont celles qui engagent le plus la $\mathrm{MDPH}$, car elles obligent les établissements médico-sociaux correspondants.

À ces orientations s'ajoutent des « préconisations », plus souples : services d'évaluation et d'orientation comme le « service appui-projet » (SAP) ou la « Prestation d'Orientation Professionnelle Spécialisée » (POPS) ; l'indication d'un " référent » comme Pôle emploi, les services spécialisés d'aide à la recherche d'emploi (Cap emploi) ou de maintien dans l'emploi (Sameth). Ces référents donnent accès à des droits qui ne sont pas du ressort de la MDPH et sur lesquels elle n'informe pas. La RQTH en elle-même ouvre relativement peu de droits, outre le fait d'être éligible au quota d'embauche : I'accès aux services spécialisés de placement ou d'aide au maintien dans l'emploi, des aides pour l'aménagement du poste de travail, un accès aux emplois aidés. Elle n'ouvre pas de droit en elle-même à une allocation financière.

Notons que deux millions de personnes relevaient de I'OETH en 2011 (dont 1,15 millions de titulaires de la RQTH). Le taux d'activité était de $44 \%$, le taux d'emploi de $35 \%$ (Amrous et alii, 2013). Parmi les travailleurs handicapés, les orientations en milieu protégé sont minoritaires. $81 \%$ des travailleurs handicapés en emploi étaient en milieu ordinaire en 2013 (ibid.). En 2014, il y avait 31500 travailleurs handicapés en atelier protégé et 117000 en Esat (Bahroumi, Chabanon, 2015).

En 2014, les demandes de RQTH représentaient $17 \%$ des demandes déposées en MDPH, I'orientation professionnelle $12 \%$ et I'AAH $18 \%$ (ibid.). 
1) Loi du 30 juin 1975 d'orientation en faveur des personnes handicapées ; loi du 10 juillet 1987 en faveur de l'emploi des travailleurs handicapés ; loi du 11 février 2005 pour l'égalité des droits et des chances, la participation et la citoyenneté des personnes handicapées.

2) Établissements et services d'aide par le travail, qui ont remplacé les CAT, Centres d'aide par le travail.

3) Association de gestion du fonds pour l'insertion des personnes handicapées (Agefiph) dans le secteur privé et Fonds pour l'Insertion des Personnes Handicapées dans la Fonction Publique (FIPHFP).

4) Commission Technique d'Orientation et de Reclassement Professionnel. Sur ces instances, voir Blanc (2004). Il existe par ailleurs des dispositifs locaux de coordination : les plans régionaux pour l'insertion des travailleurs handicapés qui ont remplacé, à partir de 2009, les plans départementaux pour l'insertion des travailleurs handicapés (Gayraud, 2009).

5) Ce temps de décision peut s'expliquer de plusieurs manières : sous-effectifs, oubli de pièces justificatives, passage par plusieurs instances d'évaluation, demande de renseignements complémentaires... Sur les questions soulevées par l'instauration d'un délai de prise de décision, voir Baudot, 2015.

À partir d'une recherche menée de 2010 à 2013 (voir encadré 2), nous nous intéressons ici aux relations entre demandeurs de la Reconnaissance de la Qualité de Travailleur Handicapé (RQTH) et Maisons Départementales des Personnes Handicapées (MDPH) et aux enjeux que ces demandes recèlent pour chacun des protagonistes. Au carrefour des politiques médico-sociales et des politiques de l'emploi, la figure du travailleur handicapé est en effet particulièrement exemplaire des conflits entre l' "injonction à l'autonomie ", présente dans les politiques sociales (Duvoux, 2009) et le "droit au non-travail ", traditionnellement attaché au handicap (Castel, 1995).

\section{Encadré 2 : Méthodologie}

L'article s'appuie sur une recherche menée avec Vincent Caradec et Jean-Sébastien Eideliman, au sein du Centre de Recherche «Individus, Épreuves, Sociétés » (CeRIES), financée par la Mission Recherche (MiRe) de la Direction de la recherche, des études, de l'évaluation et des statistiques (Drees) et la Caisse nationale de solidarité pour l'autonomie (CNSA). Pour comprendre comment la Reconnaissance de la Qualité de Travailleur Handicapé (RQTH) est investie ou non tant par les demandeurs que par les professionnels qui traitent leurs demandes, nous avons enquêté dans deux départements. Nous avons choisi deux territoires assez différents, avec un département de province plutôt populaire (département $A$ ), et un département plus aisé, situé en région parisienne (département B). Ce choix permet d'étudier des situations locales particulièrement contrastées (Bertrand et al., 2014).

Notre enquête était composée de trois volets : 40 observations de commissions traitant de l'insertion professionnelle dans les MDPH, complétées par 15 entretiens d'explicitation avec des professionnels participant à ces commissions; la saisie anonymisée de 451 dossiers de demande de RQTH ; enfin, 38 entretiens avec des demandeurs de la RQTH. De plus, nous avons pu accéder, dans le département $A$, à une base de données regroupant les demandes de RQTH ayant fait l'objet d'une décision en 2010. L'enquête de terrain s'est déroulée de début 2010 à mi-2011. 
Le recueil des données a été légèrement différent dans les deux départements. Dans le département $A$, nous avons observé les différentes équipes pluridisciplinaires traitant de l'insertion professionnelle et des réunions de la Commissions des droits et de l'autonomie des personnes handicapées (CDAPH). Dans le département B, l'organisation de la MDPH étant fortement décentralisée, nous avons enquêté dans trois "coordinations locales", en choisissant des territoires qui maximisent les contrastes du département entre des zones plus ou moins favorisées, urbaines ou plus rurales. Nous y avons observé les équipes pluridisciplinaires chargées de l'insertion professionnelle, ainsi que les réunions de CDAPH traitant des dossiers examinés dans ces équipes.

Concernant les saisies de dossiers, dans le département $A$, nous avons procédé à un échantillonnage à partir de la base de données dont nous disposions. Nous avons sélectionné et saisi des dossiers venant de tout le département. Nous n'avons pas pu opérer de la même manière dans le département $B$, où l'informatisation des demandes était moins avancée. Les coordinations locales nous ont donné accès à des dossiers de demandeurs de la RQTH pour nos saisies de données.

Pour le recrutement des interviewés, dans le département $A$, nous avons délimité trois souspopulations de la base de données : les demandeurs ayant obtenu la RQTH sans percevoir I'AAH, les demandeurs ayant obtenu la RQTH et percevant l'AAH (1), les demandeurs n'ayant pas obtenu la RQTH (ni l'AAH). Nous avons tiré des demandeurs au hasard dans ces souspopulations, à qui nous avons envoyé un courrier et que nous avons ensuite contactés par téléphone. Les personnes enquêtées sont réparties dans tout le département. Dans le département $B$, les coordinations locales nous ont transmis des coordonnées de demandeurs de la RQTH, que nous avons contactés de la même manière. La diversité des dossiers était donc moins bien contrôlée, mais semble acceptable au regard des entretiens réalisés (2). Toutefois, ce mode de recrutement ne nous a pas permis de rencontrer des personnes dont la demande de RQTH a été refusée. Dans les deux départements, les demandeurs que nous avons rencontrés avaient reçu une réponse de la MDPH depuis moins d'un an.

Notre grille d'entretien était structurée autour de trois moments : avant la demande ; au moment du dépôt de la demande et de son instruction ; après la réponse de la MDPH. Elle se terminait sur des avis plus généraux et des données biographiques. Nous demandions en fin d'entretien l'autorisation de consulter les dossiers de demande. La plupart des personnes ont accepté, ce qui a permis de mieux reconstituer le parcours institutionnel de la demande et d'accéder, le cas échéant, au " projet de vie » y figurant. L'annexe en version électronique de I'article synthétise certains éléments sur la situation des demandeurs rencontrés.

(1) Le recrutement d'allocataires de l'AAH peut étonner en raison de l'éloignement supposé de ces personnes de l'emploi. II se justifie pour deux raisons : d'une part, il s'agissait de comprendre le sens que revêtait la RQTH, y compris dans des situations où elle pouvait être moins importante - mais où elle était attribuée ; d'autre part, il existe un réel intérêt public pour une (re)mise au travail des allocataires de I'AAH (Bertrand, 2013). Sur quatorze allocataires rencontrés, quatre étaient en formation, trois cherchaient du travail, deux travaillaient en milieu protégé, un occupait un emploi à temps partiel dans le milieu ordinaire. Quatre seulement étaient inactifs. Pour des chiffres nationaux, voir Barhoumi, 2015.

(2) Perception ou non de l'AAH, âge, rapport à l'emploi, type de déficience notamment (cf. annexe électronique). 
L'orientation et le reclassement des demandeurs de la RQTH sont ainsi au centre de préoccupations divergentes. Les débats autour de la création d'une prestation de compensation du handicap, en 2005, avaient accordé une grande place au " projet de vie » qu'exprimeraient des demandeurs lourdement handicapés. Ce " projet de vie " serait une manifestation de l'autonomie de la personne qui, même dans une situation de grande dépendance, doit pouvoir exprimer ses choix personnels (Winance, 2007). Dans le champ de l'insertion professionnelle des travailleurs handicapés (voir encadré 1), dont les déficiences sont en général moins importantes que celles des bénéficiaires de la prestation de compensation, le sens du " projet de vie " ${ }^{2}$ que le demandeur peut écrire est assez ambiguë. La tentation est grande, pour les administrations centrales notamment, d'en faire l'amorce d'un projet d'abord professionnel.

En croisant les réflexions en termes d'autonomie et de parcours de vie dans le cadre des politiques du handicap nous voudrions ici aborder l'influence de la RQTH sur les parcours de vie des personnes et le travail des MDPH (partie 1), puis nous intéresser à ce qui, dans ces parcours, échappe à l'emprise institutionnelle ou relève a contrario d'une remise de soi aux institutions (partie 2).

\section{Quelle influence sur les parcours de vie?}

Dans cette première partie, nous allons nous interroger tout d'abord sur l'influence de la RQTH sur les parcours de vie des demandeurs que nous avons interrogés (1.1), avant d'étudier l'action des MDPH sur leurs projets (1.2).

\subsection{Une influence difficile à discerner}

La RQTH a des effets divers sur les parcours, que nous voudrions préciser ici à partir du point de vue des demandeurs rencontrés en entretien. Nous expliciterons chaque attitude par des exemples, l'annexe donnant une vision de l'ensemble des situations.

Parmi les personnes rencontrées, on trouve peu de reconversions réussies et stables ( $n^{\circ}$ A12, A20, B7 dans l'annexe de la version électronique de l'article). C'est le cas pour Richard Odzinski ${ }^{3}$ et Christian Vanheck (tous deux dans le département A). Richard Odzinski, 56 ans, a travaillé 37 ans comme agent de maîtrise et concierge dans

\footnotetext{
2. Concrètement, la quatrième page du formulaire de demande est intitulée " expression des attentes et des besoins de la personne (projet de vie) » et laisse une vingtaine de lignes pour écrire celui-ci. Dans la mesure où cet écrit est plus vu dans la pratique comme une pièce du dossier, un «document" (Jaeger, 2009) quelque peu réifié, nous emploierons systématiquement les guillemets pour désigner l'objet " projet de vie ", à distinguer des " projets", au sens large, de la personne.

3. Les noms indiqués ici sont des pseudonymes.
} 
une usine agro-alimentaire. Suite à une délocalisation, il perd son emploi et son logement de fonction. Quelques mois après son licenciement économique, il est victime d'un accident vasculaire cérébral, dont il garde des séquelles. Il retrouve un logement social au titre du handicap, et un emploi à temps partiel grâce à la RQTH, à la cantine d'un lycée. La RQTH a ici joué un rôle décisif pour le reclassement d'un homme en fin de carrière, relativement peu qualifié. Son épouse, qui travaillait dans la même usine et n'a pas de problème de santé, n'a pas retrouvé d'emploi stable. Christian Vanheck, 40 ans, a quant à lui travaillé plusieurs années comme carreleur jusqu'à un accident de travail à l'âge de 32 ans. Il en a gardé des séquelles : il a des difficultés à plier le genou, qui reste douloureux. Il a été trois ans en arrêt maladie. À l'issue de cet arrêt, il obtient la RQTH pour cinq ans et est orienté vers Cap Emploi. On lui propose rapidement une formation de deux mois et un recrutement comme magasinier en contrat à durée indéterminée dans un hypermarché.

Certains demandeurs ont également pu négocier une relative adaptation de leur poste de travail ( $\left.n^{\circ} \mathrm{A} 1, \mathrm{~A} 6, \mathrm{~B} 4\right)^{4}$, d'autres sont en formation ou vont entrer en formation au moment de l'entretien ( $\mathrm{n}^{\circ} \mathrm{A} 4, \mathrm{~A} 7, \mathrm{~A} 8, \mathrm{~A} 9, \mathrm{~A} 11$, B9, B15). Un petit nombre travaille en ESAT ( $\left.n^{\circ} \mathrm{A} 13, \mathrm{~B} 5, \mathrm{~B} 10, \mathrm{~B} 16\right)$. Mais pour un nombre non négligeable, la RQTH a eu peu d'effets ( $\left.n^{\circ} A 2, B 2, B 3, B 11\right)$ ou des effets non reconnus par la personne ou jugés insuffisants ( ${ }^{\circ}$ A3, B1, B6, B 16, B 17) $)^{5}$. Pour ces demandeurs, malgré les attentes qu’ils y plaçaient, parfois même après une formation, la RQTH n'a conféré qu'une faible protection et n'a pas permis d'accéder à un emploi stable. C'est le cas pour Ali Klamer, 39 ans, qui était peintre en bâtiment en Algérie avant d'émigrer en France (dans le département A). Ali a des problèmes de santé qui ne semblent pas tous très bien cernés : outre de l'asthme et des problèmes de dos, une certaine souffrance psychique et peut-être des problèmes neurologiques. Il est intérimaire par l'intermédiaire d'une agence dédiée aux travailleurs handicapés. Les missions semblent très fluctuantes, la durée maximale a été d'un mois et demi. Elles sont parfois peu adaptées : il a effectué des missions comme peintre, mais l'exposition aux poussières et aux produits chimiques le gène beaucoup en raison de son asthme.

Ali Klamer avait obtenu une RQTH pour cinq ans en 2005. Il espérait alors que cette mesure lui permettrait d'accéder à un emploi adapté. Aujourd'hui, il est très amer : il effectue les mêmes tâches que des ouvriers sans RQTH et n'a pas trouvé d'emploi stable. Il ne demande le renouvellement de sa RQTH que parce que l'agence l'exige. Il y a ici un certain paradoxe : il n'a obtenu des missions, qu’il juge inadaptées, que par cette agence d'intérim spécialisée, ses démarches dans les autres agences n'ayant pas abouti. Sa RQTH

4. Dans ce paragraphe et dans la note suivante, les numéros se rapportent à l'annexe qui figure dans la version électronique de l'article.

5. Pour d'autres enfin, la RQTH suscitait peu d'attentes, et l'acquisition du statut ou son refus suscitent peu de réactions ( $\mathrm{N}^{\circ}$ A 5, A10, A 14, A 15, A 16, A 19, B 8, B 12, B 13, B14). Parmi les personnes pour lesquelles la RQTH a été refusée, plusieurs ont déposé un recours (A 17, A 18, A20) et une autre pense déposer une nouvelle demande (A 21). 
expirant, il a donc en fait réellement besoin de la nouvelle attestation pour pouvoir travailler. La RQTH l'a objectivement aidé à trouver du travail, mais un travail très précaire et inadapté.

Chez cet homme, le travail est vu comme une obligation quasi vitale, qui surclasse les problèmes de santé. Ali vit de forts tiraillements entre une obligation de travailler s'il veut survivre et ne pas être expulsé, et la dureté des conditions de réalisation de cette obligation, compte tenu des emplois qu'on lui propose. La RQTH lui apporte une aide ambiguë, mais dont il ne peut concrètement se passer. Néanmoins, sa trajectoire est relativement ascendante : il a pu trouver un logement social par le $1 \%$ logement, sa situation administrative se stabilise, il s'est marié, est sorti de l'alcool et apparemment de ses idées noires.

Par ailleurs, pour certains demandeurs, le cumul de difficultés et la diversité des interlocuteurs institutionnels permettent difficilement d'isoler le handicap et l'influence marginale de la MDPH dans une situation confuse. On peut évoquer ici la situation d'Anne Banski, 49 ans, qui était aide-soignante à domicile dans le département $\mathrm{B}$ et a eu un accident du travail en 2005 en portant quelqu'un. Elle est opérée du dos, mais une hernie subsiste. Elle ne peut rester longtemps ni debout, ni assise et doit alterner les positions. Elle est licenciée pour inaptitude en août 2006. À la fin de son arrêt maladie, reconnue travailleuse handicapée, elle suit une formation en BEP agent administratif, d'octobre 2006 à juillet 2007. Elle effectue deux stages dans des hôpitaux proches, mais n'est pas embauchée. Elle pense à un reclassement comme secrétaire médicale, mais la formation impliquerait deux heures et demie de trajet, inimaginables dans son état. Anne est suivie par Cap emploi depuis un an. Sa conseillère lui propose des offres d'emploi, elle a eu des entretiens, mais rien n'a abouti. Le licenciement d'Anne Banski, suivi de sa reconnaissance comme travailleur handicapé, a été concomitant à un divorce, la mise en place d'un dossier de surendettement, des déménagements et ré-emménagements.

Ces situations où ce qui amène à déposer une demande est associé à des problèmes très divers sont fréquentes et il est malaisé de démêler ce qui ressort de la médecine du travail, d'un conflit avec l'employeur, de la sécurité sociale, de la MDPH et des diverses difficultés qui peuvent apparaître avec la déstabilisation de l'emploi : problèmes de logement, de couple, endettement, papiers... La RQTH n'est alors qu'un des éléments dans une situation personnelle difficile, qui souvent apporte peu de solutions en elle-même aux problèmes les plus graves.

\subsection{Agir sur les projets}

Pour certains demandeurs, les problèmes de santé sont tels qu'ils ne peuvent plus exercer leur métier. Pour retrouver un emploi, quand cela est encore possible, une reconversion est nécessaire. Elle peut être possible rapidement ou faire l'objet d'une formation plus longue. Dans ces cas, la MDPH peut notamment ouvrir le droit à un stage en Centre 
de Rééducation Professionnelle (CRP), pendant lequel le stagiaire perçoit des revenus proches de son salaire antérieur ${ }^{6}$.

La réorientation ou le reclassement d'un travailleur handicapé met en jeu les aspirations de la personne, qui peuvent être plus ou moins claires, ses incapacités et ses capacités préservées, ses compétences, sa mobilité, mais aussi l'offre institutionnelle disponible et les critères légaux d'éligibilité. Face à une demande d'orientation professionnelle, les équipes des MDPH s'interrogent sur l'adéquation entre les postes ou formations envisagées et les aptitudes du demandeur, sa formation ou son travail antérieur, bref questionnent la " cohérence " du projet professionnel. Des critères comme l'âge sont parfois mis en avant : on hésitera plus à octroyer une formation coûteuse à une personne en fin de carrière qu'à un jeune demandeur. Pour rendre plus explicites les critères mis en ouvre pour prendre une décision, nous allons nous appuyer sur trois situations. La première est évoquée lors d'un entretien avec la référente insertion professionnelle du département $B$, soit la personne plus particulièrement chargée des questions d'emploi au sein de la MDPH.

"Enquêteur. - Et quelqu'un qui vous demande une formation en CRP directement, par exemple dans son "projet de vie": "je voudrais faire telle formation dans tel centre"; vous réagissez comment? Enfin, vous faites comment?

Référente Insertion professionnelle.- Déjà, on regarde si elle relève du reclassement professionnel, sinon bah on lui dit: "non, désolé, il faudra chercher dans l'offre de formation du droit commun"; si elle relève du reclassement, dans ces cas-là, nous pouvons proposer une évaluation POPS [prestation d'orientation professionnelle spécialisée] pour vérifier qu'elle a le niveau requis et que cette formation lui convient. Parce qu'en fait, lorsque nous notifions un accord de formation, le CRP ne peut pas sy opposer. Donc, il faut vraiment être sûr, avoir pris les garanties, vérifier que la personne a le niveau requis et qu'elle soit... et qu'il n'y ait pas de contre-indications médicales à suivre cette formation. "

La référente insertion professionnelle déroule ici de manière précise la procédure habituelle : tout d'abord, vérifier si le demandeur satisfait aux critères légaux (notamment ne plus être apte à exercer sa profession d'origine, ne plus avoir de contrat de travail). Ensuite évaluer la validité de la demande. Il existe pour cela plusieurs dispositifs où le demandeur va rencontrer un professionnel, en lien avec l'équipe pluridisciplinaire de la MDPH, qui va "valider " le projet. Ici, la référente pense à une mesure fréquemment sollicitée, la POPS, réalisée par un psychologue du travail de Pôle emploi. La référente mentionne ici des critères de niveau de formation et d'aptitude médicale. Son hésitation finale (« et qu'elle soit... ») porte peut-être sur des critères plus subjectifs comme la motivation ou l'âge. Des éléments plus extérieurs comme l'état du marché du travail et les débouchés éventuels des

6. Rémunération calculée sur une moyenne des salaires perçus lors des six derniers mois d'activité avec un plancher de 652,02 € et un plafond de 1932,52 €. Si la personne ne remplit pas les conditions d'activité (six mois d'activité salariée au cours des douze derniers mois), une rémunération forfaitaire de 652,02 € est versée. Ces rémunérations sont versées par l'Agence de services et de paiement. 
formations demandées interviennent aussi parfois. Pour la MDPH, l'enjeu est clairement exprimé : sa décision d'orientation s'impose au centre de rééducation professionnelle. Les places sont limitées et il y a également un enjeu financier dans la mesure où les formations sont rémunérées. Pour la MDPH, "il faut vraiment être sûr » de sa décision.

Pour la plupart des demandeurs, le "projet de vie » n'est pas immédiatement aussi précis et les pistes de reclassement sont floues. S’ils sont éligibles au reclassement professionnel, ils peuvent intégrer un stage de pré-orientation de trois mois dans un centre de rééducation professionnelle, afin de dégager des pistes de reconversion.

Ces stages de pré-orientation sont mentionnés dans l'extrait d'observation suivant. L'observation porte sur une réunion d'une équipe pluridisciplinaire, qui étudie des demandes de RQTH et d'orientation professionnelle à la MDPH du département A. Autour de la référente insertion professionnelle et d'un médecin, la réunion rassemble différents partenaires de la MDPH. Dans la première situation évoquée, le demandeur sort d'un stage de pré-orientation ; dans la seconde, l'envoi en pré-orientation est préconisé ${ }^{7}$ :

- La demande émane d'un homme de 35 ans, ancien du bâtiment "qui a explosé son épaule» (selon le médecin de l'équipe). Il sort d'un stage de pré-orientation qui a débouché sur une préconisation de remise à niveau de six mois en vue d'une formation de technicien en assistance informatique. Mais les membres de l'équipe pluridisciplinaire jugent le projet irréaliste ; d'une part, il semble que ce soit un "projet par défaut " dont il n'a pas su expliquer la motivation ; d'autre part, il a des «capacités logiques limitées ». Le médecin déclare que "médicalement, il peut le faire. Mais son projet n'est pas cohérent ". Il est décidé d'attendre la fin du stage de remise à niveau, puis de revoir son projet professionnel. Quelqu'un dit: "C'est comme un adolescent qui doit chercher son orientation".

- Le même jour, l'équipe examine la demande d'un homme de 31 ans, qui souffre d'une hernie discale et veut lui aussi se former en informatique : "Avec un CAP, ouh là !" s'exclame le représentant de Pôle Emploi, "en informatique, il faut au minimum bac + 2, et même avec on a du mal à trouver du boulot». Le décalage entre son niveau scolaire et la formation visée est souligné et l'équipe s'accorde sur un envoi en stage de pré-orientation afin de «l'ouvrir sur autre chose ».

Dans la première situation, c'est la motivation et peut-être les capacités du demandeur qui posent question ; dans la seconde, c'est l'inadéquation entre les compétences scolaires et le niveau attendu sur le marché du travail qui suscitent des doutes, sur fond de critiques récurrentes envers une formation en informatique jugée insuffisante. Dans les deux cas, l'équipe ne se prononce pas pour un refus net, mais espère une évolution du projet après un stage de remise à niveau d'un côté, un stage de pré-orientation de l'autre.

7. Pour ces deux situations, les notes sont ici celles d'un collègue participant à la recherche, pour une séance à laquelle j'ai également assisté. 
Loin d'un acquiescement d'emblée aux projets des demandeurs, le reclassement des demandeurs fait donc l'objet d'une forme de négociation à distance. L'équipe pluridisciplinaire ne rencontre pas en personne les demandeurs, mais par ses refus ou réorientations, l'envoi vers l'une ou l'autre des instances d'orientation ou de validation et les retours qui lui sont faits, elle agit sur les projets, les retravaille avant de les valider le cas échéant. Même si la MDPH ou ses partenaires n'imposent pas de solution, la relation reste donc asymétrique (Béguin, Bertrand, 2012).

Dans cette partie, nous avons souhaité donner un cadre aux relations entre demandeurs et MDPH : d'un côté, une influence parfois réelle, mais souvent indiscernable pour les demandeurs ; de l'autre, une négociation sur les projets de reconversion. Nous poursuivons cette réflexion dans une deuxième partie en soulevant deux problèmes supplémentaires : la difficulté des MDPH face aux initiatives des demandeurs, et les phénomènes de remise de soi à l'institution.

\section{Les relations à la MDPH, entre autonomie et remise de soi}

\subsection{L'autonomie des usagers face aux fonctionnements de la MDPH}

Pour bien des demandeurs, la MDPH représente une ressource peu fiable, qui crée des attentes et les frustre à la fois. Son fonctionnement leur paraît opaque, avec parfois des désajustements difficiles. Les temporalités sont très différentes entre le temps institutionnel, où l'instruction peut prendre plusieurs mois, et le temps des demandeurs, marqué tour à tour par un étirement maussade de l'attente et par l'urgence de se sortir d'une situation difficile. Cela est plus visible chez les personnes dont la demande coïncide avec des ruptures dans le parcours professionnel : l'attente de la formation de rééducation professionnelle promise peut être longue, avec des effets parfois désastreux. L'exemple suivant, d'une jeune femme rencontrée en entretien dans le département $B$, en fournit une illustration.

Ana Ruaro Dos Santos est une jeune femme de 25 ans, qui a longtemps été coiffeuse et réside, au moment de l'entretien au printemps 2011, dans la banlieue parisienne, à environ une heure de Paris. Elle est devenue allergique à un produit très volatil et d'emploi fréquent en salon. En octobre 2009, elle est mise en arrêt maladie et adressée à un centre d'allergologie. Ce centre monte le dossier RQTH, envoyé en décembre 2009. Devenue incapable de travailler dans un salon de coiffure, Ana est finalement licenciée pour inaptitude en avril 2010. Elle s'inscrit alors à la Mission locale. En dehors du salon, elle ne souffre plus de problèmes allergiques. Après plusieurs stages, elle décide de se reconvertir dans la pâtisserie et cherche alors une formation. 
En octobre 2010, sans avoir eu de nouvelles de sa demande de RQTH depuis décembre 2009, elle est convoquée pour une " prestation d'orientation professionnelle spécialisée ». Elle apprend alors qu'elle bénéficie de la RQTH pour trois ans par une décision du 10 juin 2010. Elle évoque son projet de reconversion. La psychologue du travail trouve une formation en pâtisserie dans un centre de rééducation professionnelle éloigné. Une commission statuera sur cette demande le 30 novembre. Mais après cette date, hormis une demande de test d'une éventuelle allergie aux ingrédients employés en pâtisserie, rien ne se passe. Ana doit pourtant recevoir une notification pour quatre mois de remise à niveau et onze mois de formation, à partir du 8 mars 2011.

Sans nouvelles, elle " harcèle " la MDPH et apprend que la commission n'aura lieu que le 20 janvier. On lui confirme qu' elle aura accès à cette formation, elle interrompt donc ses recherches d'autres modalités de formation. Mais elle n'est toujours pas formellement inscrite au CRP. Vers la mi-février, elle appelle le CRP qui lui dit ne pas avoir reçu son dossier. Elle finit par se déplacer à la $\mathrm{MDPH}$, où un agent retrouve la notification manquante. Mais à quelques jours du début du stage, il n’y a plus de place pour la remise à niveau de quatre mois. Après des démarches infructueuses pour suivre cette remise à niveau ailleurs, sa conseillère de la Mission locale suggère qu'elle pourrait rentrer directement en formation en août, sans remise à niveau. Ana retourne à la $\mathrm{MDPH}$, l'agent recontacte le CRP, qui est d'accord, et modifie la notification en conséquence. Ana pourra intégrer, en août 2011, le CRP souhaité.

En quelques mois, Ana est passée d'une relative stabilité financière et affective à une période de grande précarité. La baisse de ressources consécutive à son licenciement l'amène à s'endetter auprès de ses proches. Rapidement, elle et son compagnon sont contraints de quitter l'appartement qu'ils occupaient faute de moyens financiers. Ils logent chez les parents de l'un puis de l'autre, avant de vivre chacun chez leurs parents respectifs. Le couple se sépare et se réconcilie plusieurs fois. Au moment de l'entretien, ils ont repris un logement ensemble.

Les parents d'Ana ont déménagé il y a quelques années dans une ville où elle n'a pas grandi. Chez eux, elle vit une espèce d'assignation à résidence : ses parents ne sont pas là en journée, les sorties à Paris ou pour voir ses amies coûtent cher, elle ne connaît personne sur place et reste " enfermée " à la maison. Elle est assez désœuvrée, son moral est en dents de scie, meilleur quand elle a un stage ou qu'elle prend des missions d'intérim que lui confie ponctuellement un ami.

À bien des égards, Ana est une cible idéale pour les institutions de l'insertion professionnelle, avec bien des atouts : jeune âge, handicap très localisé, mobilité, dynamisme, recherche active d'une reconversion, gestion autonome des documents administratifs. Elle a une remarquable capacité à rebondir après "les coups durs ". Pourtant, le traitement de ses demandes est particulièrement complexe et lent. Elle est contrainte, pour reprendre une expression d'Ulrich Beck, de « résoudre sur le plan biographique les contra- 
dictions du système» (Beck, 2003, p. 293), ce qui a des effets désastreux sur son couple, sa situation financière et son moral.

Si le fonctionnement des MDPH et les droits auxquels elles donnent accès échappent au demandeur, symétriquement, certains demandeurs échappent à l'emprise que les MDPH pourraient avoir sur leurs biographies. Par exemple, il sera difficile de contraindre quelqu'un qui ne veut pas quitter son ancien métier et qui continue parfois de l'exercer, malgré les contre-indications. L'emprise institutionnelle pourrait paraitre plus forte quand le travailleur handicapé demande une formation, mais il n'est pas rare qu'il préfère trouver du travail par ses propres moyens plutôt que suivre le parcours de formation proposé ou qu'il trouve un emploi dans un autre domaine à l'issue de la formation accordée.

Le cas de Patrick Vermauld, 23 ans, vivant dans le département A, constitue ainsi un bon exemple d'une progression par tâtonnements, de recours aux institutions du handicap et d'hésitations par rapport aux réponses proposées, de recherches d'emploi par soi-même. Patrick est porteur d'une maladie rare qui, parmi d'autres symptômes, entraîne chez lui une légère déficience intellectuelle. Pendant sa scolarité, ses parents ont cherché à mettre à distance les institutions liées au handicap. Mais au moment de s'insérer professionnellement, s'il parvient à trouver des emplois temporaires, notamment dans les espaces verts, les retentissements de sa maladie rendent difficile le maintien en emploi. Ses parents se résolvent à déposer une demande de RQTH, qu'il obtient avec une orientation en "atelier protégé ". Patrick intègre alors un atelier protégé, mais vit mal la confrontation à des collègues au handicap plus marqué. Il quitte l'atelier. Avec ses parents, il se démène alors pour trouver une formation qui permettrait de trouver un emploi stable. Mais il échoue aux tests pour intégrer une formation de droit commun à l'AFPA (Association nationale pour la formation professionnelle des adultes).

Patrick trouve par lui-même un CRP, dans lequel il aimerait suivre une formation $\mathrm{d}$ ' "agent d'entretien du bâtiment ». Il dépose une demande en ce sens avec l'aide de ses parents. La MDPH hésite : les tests passés à l'AFPA étaient très insuffisants, même pour une formation adaptée et Patrick bénéficiait déjà d'une orientation pour un travail en milieu protégé. Il est convoqué et, avec l'aide de ses parents, il convainc la commission, qui l'oriente vers un stage de pré-orientation de trois mois.

À l'issue de ce stage, le centre lui propose une orientation vers un CRP dans une autre région pour une formation d'ouvrier paysagiste, précédée d'une remise à niveau. La notification qu'il reçoit alors est valable plusieurs mois. Mais Patrick fait un choix différent : il reprend un travail saisonnier chez un ancien employeur pour du débroussaillage. Il harcèle même Pôle emploi avec son patron pour trouver un financement pour un " permis remorque ". Patrick n'est pas réfractaire à une entrée en CRP, mais tant qu’il trouve du travail, il pense repousser son entrée en formation. À présent, il estime avoir mûri et serait aussi prêt à retravailler en atelier protégé. 
Le parcours de Patrick est ainsi fait d'usage tactique de la RQTH, d'hésitations, de demandes de certaines mesures, de refus de certaines solutions, d'insistance pour l'accès à une formation puis de bifurcations.

\section{2 «Merci de ce que vous pourrez faire de moi » ou s'en remettre à l'institution}

La sous-partie précédente insistait sur les initiatives et les aspirations des demandeurs, et les difficultés des MDPH à les prendre en compte. Remarquons que les deux exemples fournis sont issus, pour Ana, d'un entretien et de l'observation de l'examen de sa demande en équipe pluridisciplinaire, pour Patrick, d'un entretien puis de la lecture de son dossier de demande. La relation particulière qui se noue dans l'entretien amène peut-être à mieux prendre en considération la "puissance d'agir ${ }^{8}$ de nos interlocuteurs. L'étude des dossiers de demandes, sans avoir rencontré les personnes, amène à être plus attentif à d'autres dimensions. Dans le « projet de vie » qui suit, c'est plutôt la remise de soi qui prédomine. Le texte, émaillé de nombreuses fautes, sans saut à la ligne ni ponctuation, à l'écriture malaisée, émane d'une ancienne auxiliaire de vie du département B âgée de 34 ans, qui a par ailleurs coché la case " Je souhaite être aidé(e) par un professionnel de la MDPH pour exprimer mes attentes et mes besoins ». Nous le rendons ici dans une forme en partie normée :

"Je suis fatiguée, depuis un an mes douleurs dans les jambes ne me permettent plus d'accomplir mon objectif de faire ma formation d'aide-soignante. J'ai six ans d'expérience dans le métier d'auxiliaire de vie et j'aime mon métier et je ne sais plus dans quel métier me tourner. J'ai besoin d'aide et de conseils, je ne pensais pas avoir cette souffrance dans mes deux jambes, mais la vie l'a voulu ainsi. En étant travailleur handicapé, j'aurais peut-être une activité professionnelle qui me soulagera dans mon métier et au sein de ma famille. J'ai deux enfants, une fille de dix ans et un garçon de trois ans. Aidez-moi à me reconstruire dans ma vie pour mes enfants, ils ont besoin de moi et j'ai besoin d'aide. Merci de ce que vous pourrez faire de moi."

Ce texte, malgré les difficultés d'écriture, ne manque pas de structure ni même de stratégie rhétorique. Simplement, comme dans les demandes envoyées au Fonds d'urgence sociale étudiées par Didier Fassin, il emprunte la forme de la "supplique ", "forme ancienne et conventionnelle par laquelle un sujet interpelle une autorité lointaine pour en obtenir une faveur ou une grâce » (Fassin, 2000). Loin de présenter les signes d'une intériorisation de l'injonction à l'autonomie, l'auteure cherche plutôt à faire reconnaître sa souffrance et s'en remet à l'institution. Pour reprendre la dichotomie énoncée par Yasmine Siblot, il s'agit ici plus de "demander l'aumône" que de "faire valoir ses droits" (2006).

Ces phénomènes de remise de soi ne sont pas rares, et en les considérant autrement, on en trouve la trace également dans les entretiens. Ainsi Suzanne Vanheck, rencontrée à son

8. Proposition de traduction de l'agency américaine, par Charlotte Nordmann et Jérôme Vidal, cf. Butler, 2004 . 
domicile dans le département $\mathrm{A}$, se montre à première vue revendicative par rapport aux débouchés de la « formation » qu'elle va bientôt commencer :

"J'ai été acceptée, je suis en formation. Là, on va morienter.. [...] Pour dire de morienter sur autre chose et puis c'est tout, hein. 15 ans d'agent d'entretien, vous savez, 15 ans, ça marque. Qu'est-ce que je vais faire, moi, à 54 ans? [...] Sur quoi je vais morienter? Moi, je veux bien faire la formation, de toute façon, c'est ce qu'on mia dit. [...] Mais moi, je vais leur dire, hein. Je vais leur dire, moi, "je veux bien faire votre formation si après, il n'y a pas de problème, vous morientez sur quelque chose". Mais moi, je veux un travail à la clé après la formation. Sinon, moi... Je vais lui dire : "de toute façon, moi, vous allez me faire perdre mon temps!" "

Les objurgations de Suzanne, dans la situation particulière d'un entretien à domicile, masquent mal son désarroi. Elle est étonnée qu’on lui ait proposé une formation à son âge - en fait un stage de pré-orientation. Elle n'a pas vraiment d'idée de ce qu'elle pourrait faire, à part peut-être téléconseillère, une amie lui ayant dit que le secteur embauchait des travailleurs handicapés. Elle fait également allusion à une sortie du marché du travail par une possible mise en invalidité. Si elle attend un "travail à la clé ", elle espère qu' " on va l'orienter».

Ces phénomènes de remise de soi paraissent décalés par rapport à l'insistance sur l'expression d'un " projet de vie » et l'autonomie de demandeurs " acteurs de leur prise en charge ». Pourtant, nombre de demandeurs sont dans l'incertitude quant à ce qu'il est possible de faire avec leurs aptitudes restantes, à l'évolution de leur pathologie, aux opportunités du marché du travail, aux droits qu'il est possible d'ouvrir? . Comment articuler un projet, rédiger un " projet de vie » en toute méconnaissance des possibles?

De fait, nombre de demandes ne comportent pas de " projet de vie » (45\% dans notre échantillon), et les projets écrits sont souvent flous ou ne mentionnent pas de projet professionnel ${ }^{10}$. D’une certaine manière, la multiplicité des mesures d'orientation est aussi un indice de ces difficultés.

\section{Conclusion}

Au terme de cet article, nous voudrions mettre l'accent sur certains points saillants de ce travail. Étudier une mesure comme la RQTH (Reconnaissance de la qualité de travailleur handicapé) peut paraître singulier. C'est une demande simple à évaluer, dont le taux de refus est faible (moins de $10 \%$, cf. Barhoumi \& Chabanon, 2015). Comme

9. Parfois, l'entrée dans le handicap se fait de manière brutale et inattendue. "Tout sécroule et on te demane de rédiger un "projet de vie" ", comme le résumait un proche confronté à cette épreuve.

10. À titre indicatif, sur les 223 projets de vie de notre échantillon, 25 ne contenaient pas de demande précise, 84 comportaient une demande vague et 114 une demande précise. Mais ces demandes précises peuvent aussi être très succinctes et peu informatives, comme cette forme limite du " projet de vie » indiquant simplement : "renouvellement de ma RQTH». 
on l'a vu, elle a rarement un effet spectaculaire sur les parcours des demandeurs. Elle fait peu l'objet de mobilisations associatives, hormis la "semaine pour l'emploi des personnes handicapés ${ }^{11}$. Elle est pourtant au centre d'une politique de quota d'embauches assez singulière en France et mobilisant des fonds importants. Si on y adjoint les interrogations sur l'orientation professionnelle et la question des " projets de vie ", l'image se complexifie.

Du côté des MDPH (Maisons départementales des personnes handicapées), quelle place accorder aux aspirations des demandeurs ? Jusqu'où les suivre ? Comment répondre à des demandes jugées inadaptées ? Comment travailler avec des " projets de vie " souvent inexistants, parfois trop laconiques, ou trop longs, n'évoquant pas de projet professionnel ou alors des projets de reconversion très précis, mais obsolètes ?

Pour certains demandeurs, la rédaction d'un " projet de vie " - expression un peu subjuguante - demande trop ou en dit trop peu : nombre d'entre eux attendent une aide à l'orientation, méconnaissent leurs droits ou sont submergés par d'autres problèmes plus ou moins liés au handicap. Mais beaucoup ont des projets qui dépassent largement ce qui a pu être écrit, ou ont évolué depuis la rédaction de leur "projet de vie ".

Nous ne prétendons pas avoir répondu ici à toutes ces questions. Mais nous voudrions préciser ce qui fait l'intérêt de cette contribution. Notre approche était particulière : non pas directement une étude des parcours professionnels, mais une étude des relations entre la MDPH et des personnes déposant des demandes liées à l'insertion professionnelle, dans un contexte censé être renouvelé par la prise en compte des "projets de vie ». Notre recherche qualitative permet de mettre l'accent sur ce qui est peutêtre plus opaque dans une approche quantitative : des effets de la RQTH souvent peu intelligibles - Ali Klamer aurait certainement répondu à un questionnaire que la RQTH n'avait pas eu d'effet, alors que sa situation est plus complexe -, une action à distance des MDPH sur les demandes de demandeurs, des temporalités différenciées. L'observation permet de montrer que, d'une part, les MDPH exercent une influence sur les parcours, mais que, d'autre part, les «projets de vie ", les aspirations des demandeurs ont une place réelle dans l'évaluation des situations. Les MDPH ne peuvent pas toujours imposer leurs choix et se retrouvent parfois débordées par l'autonomie des demandeurs.

Enfin, loin de répondre à des demandeurs sûrs de leurs choix et à même de les exprimer dans un "projet de vie ", les institutions travaillent quotidiennement avec des demandeurs déstabilisés, parfois en détresse. Alors que cette réalité est massive et plutôt bien identifiée par les professionnels, elle paraît être un point aveugle des discours publics sur les usagers " acteurs de leur prise en charge ".

11. Chaque troisième semaine de novembre. 


\section{Bibliographie}

Amrous N., Barhoumi M., Biausque V. (2013), "L'accès à l'emploi des personnes handicapées en 2011 ", Dares Analyse, nº6.

Astier I. (1997), Revenu minimum et souci d'insertion, Paris, Desclée de Brouwer.

Barbier J.-C. (2002), "Peut-on parler d' “activation” de la protection sociale en Europe ?", Revue française de sociologie, n 43, pp. 307-332.

Barhoumi M. (2015), "La situation professionnelle des bénéficiaires de l'allocation aux adultes handicapés ", Dares Analyse, n 36.

Barhoumi M., Chabanon L., (2015), "Emploi et chômage des personnes handicapées ", Synthèse. Stat', Dares, $\mathrm{n}^{\circ} 17$.

Baudot P.-Y. (2015), "L'invention des délais. Pourquoi l'administration doit-elle répondre dans les temps?", Politiques sociales et familiales, n 119, pp. 5-18.

Beck U. (2003), La Société du risque. Sur la voie d'une autre modernité, Paris, Flammarion.

Béguin H., Bertrand L. (2012), «Une approche critique de l'individualisation des politiques sociales : pistes de réflexion ", in Muller B., Barbier J.-C, Bresson M. (dir.), Les solidarités à l'épreuve des crises, Paris, L'Harmattan, pp. 95-111.

Bertrand, L. (2013). « Politiques sociales du handicap et politiques d'insertion : continuités, innovations, convergences ", Politiques sociales et familiales, $\mathrm{n}^{\circ} 111$, pp. 43-53.

Bertrand L., Caradec V., Eideliman J.-S. (2014), "Devenir travailleur handicapé. Enjeux individuels, frontières institutionnelles ", Sociologie, $\mathrm{n}^{\circ}$ 2, vol. 5, pp. 121-138.

Blanc A. (2004), Les handicapés au travail. Analyse sociologique d'un dispositif d'insertion professionnelle, Paris, Dunod.

Bresson M. (2004), "Les figures de l'individu dans l'intervention sociale ", in Caradec V., Martuccelli D. (dir.), Matériaux pour une sociologie de l'individu. Perspectives et débats, Villeneuve d'Asq, Presse Universitaires du septentrion, pp. 121-142.

Butler J. (2004), Le Pouvoir des mots. Politique du performatif, Paris, Éditions Amsterdam.

Castel R. (1995), Les métamorphoses de la question sociale. Une chronique du salariat, Paris, Fayard.

Dubet F. (2002), Le déclin de l'institution, Paris, Seuil.

Dubois V. (2010), La vie au guichet. Relation administrative et traitement de la misère, Paris, Economica.

Duvoux N. (2009), L'autonomie des assistés, Paris, PUF. 
Fassin D. (2000), «La supplique. Stratégies rhétoriques et constructions identitaires dans les demandes d'aide d'urgence ", Annales. Histoire, sciences sociales, 55-5, pp. 953-981.

Gayraud L. (2009), "Territorialisation de l'action publique : rôle et enjeux des coordinations territoriales dans les programmes départementaux pour l'insertion des travailleurs handicapés ", in Blanc A. (dir), L'insertion professionnelle des travailleurs handicapés, Grenoble, PUG, pp. 49-76.

Grignon C., Passeron J.-C. (1989), Le Savant et le Populaire. Misérabilisme et populisme en sociologie et en littérature, Paris, Gallimard/Seuil.

Jaeger M., (2009), "Focus - quand le législateur découvre la notion de projet de vie... », Informations sociales, $\mathrm{n}^{\circ} 156$, pp. 142-144.

Jouan M., Laugier S. (dir.) (2009), Comment penser l'autonomie ? Entre compétences et dépendances, Paris, PUF.

Lima L. (dir.) (2013), L'expertise sur autrui. L'individualisation des politiques sociales entre droit et jugements, Bruxelles, P.I.E Peter Lang.

Lipsky M. (1980), Street -Level bureaucracy: Dilemnas of the Individual in Public Services, New-York, Russel Sage Foundation.

Rosanvallon P. (1995), La nouvelle question sociale. Repenser l'État-providence, Paris, Seuil.

Siblot Y. (2006), Faire valoir ses droits au quotidien. Les services publics dans les quartiers populaires, Paris, Presses de Sciences Po.

Soulet M.-H. (2005), "Une solidarité de responsabilisation », in Ion J. (dir), Le travail social en débat[s], Paris, La Découverte, pp. 86-103.

Weller J.-M. (1999), L'État au guichet: sociologie cognitive du travail et modernisation administrative des services publics, Paris, Desclée de Brouwer.

Winance M. (2007), « Dépendance versus autonomie... De la signification et de l'imprégnation de ces notions dans les pratiques médicosociales ", Sciences sociales et santé, Vol. 25, $\mathrm{n}^{\circ}$ 4, pp. 83-91. 